\title{
Arabidopsis is susceptible to the cereal ear blight fungal pathogens Fusarium graminearum and Fusarium culmorum
}

\author{
Martin Urban, Steve Daniels ${ }^{\dagger}$, Ellie Mott ${ }^{\ddagger}$ and Kim Hammond-Kosack ${ }^{*}$ \\ Plant-Pathogen Interactions Division, Rothamsted Research, Harpenden, Herts, AL5 2JQ, UK \\ Received 17 May 2002; revised 11 September 2002; accepted 16 September 2002. \\ *For correspondence (fax +44 1582715009 ; e-mail kim.hammond-kosack@bbsrc.ac.uk). \\ ${ }^{\dagger}$ Current address: Monsanto UK Ltd, The Maris Centre, 45, Hauxton Road, Trumpington, Cambridge, CB2 2LQ, UK. \\ ${ }^{\ddagger}$ Current address: Department of Plant Sciences, University of Cambridge, Downing Street, Cambridge, CB2 3EA, UK.
}

\begin{abstract}
The fungal pathogens Fusarium graminearum and F.culmorum cause ear blight disease on cereal crops worldwide. The disease lowers both grain quality and grain safety. Disease prevalence is increasing due to changes in cropping practices and the difficulties encountered by plant breeders when trying to introgress the polygene-based resistance. The molecular basis of resistance to Fusarium ear blight in cereal species is poorly understood. This is primarily due to the large size of cereal genomes and the expensive resources required to undertake gene function studies in cereals. We therefore explored the possibility of developing various model floral infection systems that would be more amenable to experimental manipulation and high-throughput gene function studies. The floral tissues of tobacco, tomato, soybean and Arabidopsis were inoculated with Fusarium conidia and this resulted in disease symptoms on anthers, anther filaments and petals in each plant species. However, only in Arabidopsis did this initial infection then spread into the developing siliques and seeds. A survey of 236 Arabidopsis ecotypes failed to identify a single genotype that was extremely resistant or susceptible to Fusarium floral infections. Three Arabidopsis floral mutants that failed to develop anthers and/or functional pollen (i.e. agamous-1, apetala1-3 and dad1) were significantly less susceptible to Fusarium floral infection than wild type. Deoxynivalenol (DON) mycotoxin production was also detected in Fusarium-infected flowers at $>1 \mathrm{ppm}$. This novel floral pathosystem for Arabidopsis appears to be highly representative of a serious cereal crop disease.
\end{abstract}

Keywords: Fusarium ear blight, Gibberella zeae, Arabidopsis, deoxynivalenol, flower disease.

Introduction

Fusarium ear blight infections of cereal crops (wheat, maize, barley and rye) annually cause considerable losses to grain quality and safety in numerous geographical regions (Parry et al., 1995; http://www.scabusa.org). The disease is disseminated by both sexual and asexual spores released from the previous year's crop debris. Fusarium species synthesise a range of mycotoxins in planta. Those of particular concern to human health are the sesquiterpenoid epoxide trichothecenes, primarily deoxynivalenol (DON), 15-acetyl DON and nivalenol produced by Fusarium graminearum Schwabe (sexual stage Gibberella zeae (Schw.)) (Hohn et al., 1998), F. nivale and F.culmorum and fumonisins produced by F. moniliforme and F.proliferatum that are primarily a problem on maize and rice crops (Nelson et al., 1993; Ueno et al., 1970).

Fusarium infection of cereal ears occurs primarily only once per year at anthesis (flowering). For wheat, barley and rye, this occurs when the anthers extrude from the spike. This permits hyphae arising from the air-borne fungal spores to enter either via colonising the anther and then the filament or to penetrate directly into the top of the exposed ovary as each floret (flower) opens to release the three anthers (Kang and Buchenauer, 2000; Pugh, 1933). For certain wheat cultivars, if the anthers have been carefully removed by physical emasculation, then the incidence of infections is dramatically reduced (Strange and Smith, 1971). Anthers and pollen contain two components highly stimulatory to Fusarium hyphal growth and mycelium branching, namely choline and glycine betaine (Strange et al., 1974). Fusarium mycotoxins are produced shortly after hyphae invade floral tissue. These mycotoxins may or may not contribute to host tissue penetration by hyphae (Kang and Buchenauer, 1999; McCormick et al. 1998). Highly aggressive but none or very low mycotoxin 
producing Fusarium isolates can be readily recovered from infected field crops (Gang et al., 1998; Miedaner et al., 2000). Further colonisation of plant floral tissue involves a mixture of intercellular, intracellular, as well as extensive saprophytic aerial growth (Kang and Buchenauer, 2000; Pugh, 1933). Macroscopically, infected wheat, barley and rye ears prematurely turn a light brown colour and under moist conditions, masses of pink conidia are visible on the infected grains and the various outer ear tissues, i.e. glumes, lemma and palea. The disease does not cause a soft rot symptom; all infected tissues remain structurally intact. At harvest, the threshed grain is often shrivelled and has a 'tombstone appearance' (http://www.scabusa.org). Frequently, this grain is also internally infected with fungal hyphae lying quiescent beneath the seed testa until seed germination occurs. Non-invaded grains on a partially invaded ear/cob can also accumulate fungal mycotoxins (Hui et al., 1997).

In wheat, there appears to be three main sources of unrelated resistant germplasm to Fusarium ear blight: exotics from Chinese/Japanese origin, for example Sumai-3 (Anderson et al., 2001; Ban and Suenaga, 2000); exotics from Brazil, for example Frontana (Van Ginkel et al., 1996) and numerous lines from central Europe known to be of a distinct breeding lineage (http://www.scabusa.org). Interestingly, all the resistant germplasm sources confer a defence response that is both Fusarium species non-specific and race non-specific within a single Fusarium species. However, only a poor correlation exists between resistance to ear blight and to a second disease symptom, namely a stem base rot that is also of frequent occurrence in cereal crops (Parry et al., 1995). Some correlation between seedling resistance to the DON mycotoxin at the time of seed germination and resistance to ear infections has previously been reported (Buerstmayr et al., 1997; Wang and Miller, 1988). However, similar results have not been observed by others (Bill Hollins, personal communication; Paul Nicholson, personal communication).

There is a great paucity in our understanding of the molecular control of the Fusarium ear infection process of cereal crop species and the identity of the key components of the plant resistance response leading to effective pathogen containment in a species and race-non-specific manner. Some molecular information exists on the regulation of mycotoxin production, but this is limited to the details of the steps in the specific biosynthetic pathways and various regulatory genes (Hohn et al., 1998, 1999; Tag et al., 2001). Others have commenced characterisation of the defence genes induced in both resistant and susceptible wheat ears following Fusarium infection (Pritsch et al., 2001). In wheat, definitive plant molecular genetics experiments are difficult to carry out because of its large and hexaploid genome and the long plant generation time. Alternative diploid cereal model systems (i.e. rice and barley), although possibly appropriate, lack suitable resistant germplasm sources and also have long generation times. We therefore decided to explore whether a range of experimentally versatile dicotyledonous plant species could be exploited for high-throughput molecular genetic studies.

Fusarium symptomless infections have previously been noted on dicotyledonous weed species growing at the base of infected winter wheat crops in the UK. Jenkinson \& Parry (1994) were able to recover five Fusarium species from the surface-sterilised stem bases of 14 different weed species that showed no evidence of macroscopic disease symptoms. These species were F.avenaceum, F.culmorum, F.poae, F.sambucinum and F.graminearum, cited in decreasing order of recovery frequency. Almost all of the Fusarium isolates recovered from the weed species were confirmed as also pathogenic to the stem bases of wheat seedlings. There is also a second earlier report of visible F. graminearum infections on the surface of detached, ripe tomato fruits following artificial inoculation of wounds (Crozier and Boothroyd, 1959). Other Fusarium species, for example F. oxysporum are excellent invaders and colonisers of the roots and stem bases of hundreds of dicotyledonous species (Agrios, 1997). F. oxysporum primarily attacks vascular tissues in upper parts of the plant from the initial root and stem base infections. The direct infection of F. oxysporum of wounded tomato fruits has been previously reported (Di Pietro et al., 2001), but direct flower tissue infections have not.

In this paper, we describe experiments that show that the wheat-attacking fungal pathogens, F.graminearum and F. culmorum, can also attack the flowers of several dicotyledonous species including, tobacco, tomato, soybean and Arabidopsis to cause various disease symptoms. We also reveal that flowers lacking functional anthers, either by the use of physical ablation or genetic mutation, are highly resistant to Fusarium attack. From a screen of different Arabidopsis ecotype accessions, we did not recover any ecotypes that exhibited a high level of resistance to Fusarium floral infection. Through competitive ELISA experiments, we confirmed that DON mycotoxin accumulation occurs in infected Arabidopsis flowers. This novel floral pathosystem for Arabidopsis appears to be highly representative of a serious cereal crop disease. This compatible model system will now permit various follow-up molecular genetics and gene expression experiments to be performed to identify the defence signals and responses that restrict fungal hyphal colonisation in planta.

\section{Results}

\section{Establishing the floral inoculation system}

Various spray and droplet inoculation methods of flowers and leaf tissue of Arabidopsis, tobacco (N.tabacum), 
tomato and soybean plants were attempted using a conidial spore suspension. In all experiments, control wheat ear inoculations were performed in parallel with the identical solution of spores and environmental conditions. To obtain floral disease symptoms, it was important to maintain the plants post-inoculation under continuous high humidity conditions, but ensure the plant roots did not become waterlogged, which greatly perturbs normal flower development and anther dehiscence. Also, a period of darkness for a minimum of $16 \mathrm{~h}$ immediately post-inoculation was required to ensure uniform floral infection. Fine spray inoculations of open flowers were always found to be more effective than small droplets because the later were frequently lost to variable extents through droplet run-off.

Inoculation of immature flowers or puncture wounded/ epidermis stripped/unwounded leaves did not result in the invasion of plant tissue. Microscopic observation of trypan blue-lactophenol-stained tissue taken at various times post-inoculation revealed that Fusarium conidia germinate on the leaf surface in the absence of anthers. However, the growth of the germ tube extended a maximum of 5-10 spore length and thereafter growth ceased and the surface hyphae collapsed. There were no obvious attempts to penetrate leaf tissue. When leaf infection tests were performed by mixing Fusarium conidia with detached anthers, only a mass of saprophytically growing surface mycelium developed (Figure 1a). Microscopic observations of stained tissue again revealed the absence of tissue penetration by hyphae in the presence of pollen.

\section{The flower infection phenotypes on various solanaceous and leguminous plant species}

Spray inoculation of conidia into the open flowers of Arabidopsis ecotype Columbia, tobacco cv. Petit Havana, tomato cv. Moneymaker and soybean cv. A3244 resulted in the development of aerial mycelia in association with anther and anther filament tissue within 2 days (Figure 1b,c and data not shown). There was a striking similarity in the appearance of disease symptoms on the floral tissue of these various dicotyledonous species and those observed on the inoculated ear of the susceptible wheat cv. Bobwhite (Figure 1d and as reported previously for wheat (Kang and Buchenauer, 2000; Pugh, 1933). Microscopic observation of trypan blue-lactophenol-stained tissue taken 0.5, 1 and 2 days post-inoculation of Arabidopsis flowers revealed that conidia germinated and grew on the anther surface. Hyphal entry into the anthers typically coincided with anther dehiscence. Subsequently, hyphal growth was predominantly intercellular between the pollen grains inside the anther sac, but there was also some evidence of intracellular growth through the anther sac wall. Considerable intercellular hyphal colonisation occurred within the anther filament (Figure 1e). Subsequently, in Arabidopsis flowers, the visible infection proceeded into the petals, ovary, sepal and peduncle tissue before reaching the main stem within another 2 or 3 days. By day 5 post-infection, the main stem within the flower head had dried and constricted and turned brown or grey. Necking over of the flower head at the point of stem invasion was frequently seen and the developing siliques exhibited grey or brown necrotic symptoms and appeared slightly shrivelled, both above and below the point of stem invasion (Figure 1b). Microscopic observations revealed both inter- and intracellular hypha colonisation of stem and silique tissue (Figure $1 \mathrm{f}, \mathrm{g}$ ) and colonisation of the immature seeds within infected siliques (Figure 1h,i). A considerable amount of aerial saprophytic mycelium extended above the surface of all the colonised plant tissues (Figure $1 \mathrm{~b}, \mathrm{~g}$ ). Over the comparable 2-5 days postinoculation in wheat ears, the infection had spread beyond the colonisation of the extruded anthers into the corresponding spikelet and caused the outer glumes, lemma and palea tissues to turn pale brown. A mass of aerial hyphae extending 1-2 $\mathrm{mm}$ from the surface of the glumes was also visible (Figure 1d). By day 10 post-inoculation, the disease symptoms on Arabidopsis were maximal and the Fusarium infection did not spread down the main stem to invade the base of the plant. The formation of pink conidial masses were also evident on heavily infected floral tissue from day 6 onwards (Figure 1j). In wheat cv. Bobwhite, between 10 and 15 days are required for Fusarium to colonise the entire ear. Usually, these infections continue into the adjacent stem tissue, but these do not extend below the junction of the flag leaf. Pink conidial masses form day 12 onwards on infected spikelets and the entire ear senesced prematurely and turns pale brown by day 20 (Figure 1d).

When tobacco flowers were inoculated, a mass of aerial mycelium accumulated only in association with anthers by 2 days post-inoculation. Within another 2 days, the complete inside of the flower trumpet was filled with white mycelium (Figure 1c). This infection did not prevent the normal flower fertilisation process because when the flower petals and anthers senesced and dropped off, both macroscopic and microscopic examination revealed that the immature green seed pod below had not been invaded (Figure 1c). In tomato flowers, a similar infection phenotype was observed to that of tobacco. Due to the extreme waxyness of the anther cone of tomato flowers, a single anther was carefully removed with fine tweezers at the yellow or green stage and a droplet containing conidia was placed inside the remaining anther cone. Abundant aerial mycelium was evident with 2-3 days, but this did not affect the normal fertilisation process and green uninfected tomato fruits formed from each inoculated flower and the fruit calyx remained healthy. Spray inoculations of soybean flowers resulted in the colonisation of anthers and anther 

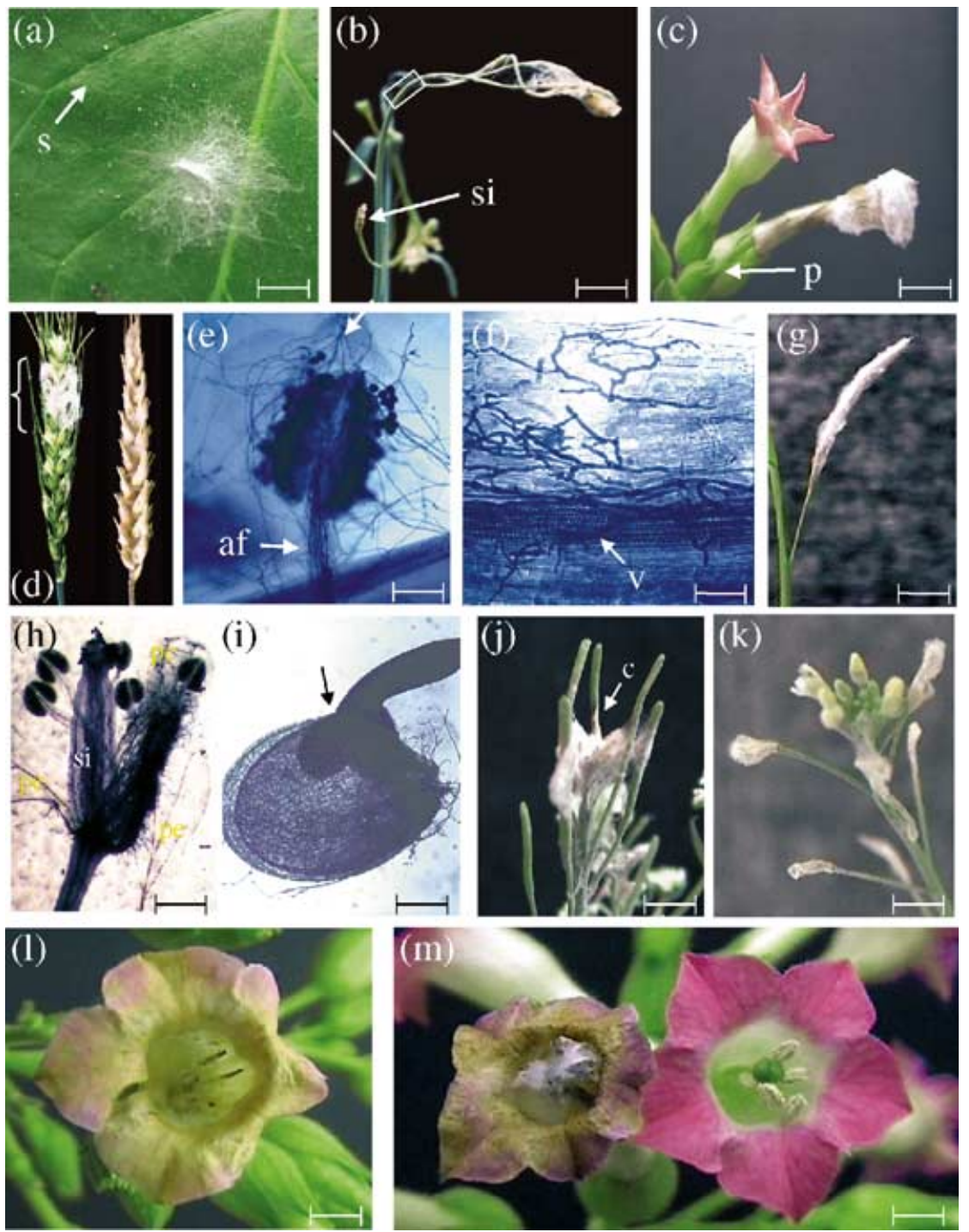

Figure 1. Various macroscopic and microscopic aspects of the Fusarium graminearum and F.culmorum disease phenotypes on floral and non-floral tissue of Arabidopsis, tobacco and wheat.

(a) Superficial growth of $F$.graminearum mycelium extending from a detached anther over the surface of a tobacco leaf. In contrast, at the leaf position indicated by S, a droplet of Fusarium conidia was placed and no hyphal growth is visible. Photograph taken 4 days post-inoculation.

(b) Arabidopsis floral tissue 5 days after spray inoculation with F. graminearum conidia. Extensive superfical mycelium covers the apical flowers and disease symptoms are evident on newly formed siliques (si). The white square indicates the stem tissue region examined by trypan blue-lactophenol staining and shown in panel (f).

(c) Tobacco flowers 4 days after spray inoculation with F. graminearum conidia. The petals are covered with mycelium and have senesced prematurely. At the base of the infected flower, the developing seed pod (p) remains disease-free.

(d) Wheat ears inoculated at anthesis and photographed at either 4 days (left) or 20 days (right) post-inoculation. Superficial mycelium is located at day 4 only where anthers were extruding from wheat florets. This region of the ear is highlighted by a white bracket. By 20 days post-inoculation, the entire wheat ear has senesced prematurely and turned brown.

$(e, f, h, i)$ Photomicrograph taken of whole tissue mounts stained with lactophenol-typhan blue.

(e) Arabidopsis anther sacs and anther filaments (af) enveloped in F. graminearum mycelium, 2 days after inoculation of flowers with conidia. Both intercellular and intracellular fungal hyphal colonisation has occurred in both anther sac and filament tissues.

(f) Stem tissue sampled just below the point of visible infection highlighted in panel (b). Both intercellular and intracellular hyphal growth is evident in the parenchyma tissue. The host cells in close contact with the Fusarium mycelium have not accumulated the trypan blue stain and are therefore still alive (Keogh et al., 1980). V, vascular tissue.

(g) An older Arabidopsis silique entirely covered with aerial mycelium, photographed 5 days after F. graminearum inoculation.

(h) A single Arabidopsis flower, 5 days after inoculation with F. graminearum. Extensive hyphal colonisation is present in both petal (pe) and silique tissues (si). (i) An Arabidopsis seed dissected from the Fusarium-infected silique shown in panel (h). Fungal hyphae are seen extending over the surface of the seed testa. The arrow indicates the suspensor cell connecting the seed to the main vascular tissue in the silique.

(j) Arabidopsis ecotype Landsberg (La-er), 7 days after inoculation with F.culmorum conidia. Extensive mycelium is present at the flower apex and on the siliques. Pink asexual conidial sporulation is also visible (c). 
filaments, but these infections never extend into the developing pods (data not shown).

Of the four dicotyledonous plant species tested, the disease symptoms forming post-Fusarium-infection were most extensive on Arabidopsis floral tissue, causing the developing seeds and stem tissue to become infected. The infections on tomato, tobacco and soybean flowers, although resulting in the formation of extensive aerial mycelium, did not proceed beyond floral tissues and did not cause seed infections.

\section{The flower infection phenotype are different between the} Arabidopsis ecotypes Columbia and Landsberg erecta

The research opportunities afforded by the Arabidopsis ecotypes Columbia Col-O and Landsberg erecta (La-er) are enormous (http://nasc.nott.ac.uk; http://arabidopsis.org). Therefore, we examined in detail whether there were differences in their Fusarium infection phenotypes. The initial inoculation experiments (described above) had already revealed considerable variation in the disease symptom phenotype manifested on the various floral tissues. Therefore, to assess quantitatively the degree of floral invasion, the infection process was divided into three subcomponent parts based upon organ type, i.e. flower infection (F) comprising of open flowers and closed buds, new siliques (NS) formed during the infection time course and the older siliques (OS) already present at the time of inoculation. Within each subcomponent, various degrees of infection were recognised based on macroscopic symptom assessments and follow-up microscopic observation of stained material (Table 1). The final Fusarium-Arabidopsis disease value (the FAD value) was calculated by adding the three subcomponent scores, i.e. individual $\mathrm{F}+\mathrm{NS}+\mathrm{OS}$ scores $=F A D$ value.

Interestingly, the La-er plants exhibited a statistically significant higher level of floral tissue disease than Col-O plants. The mean FAD value for La-er was 10.7 compared with 6.6 for Col-O when examined over 11 separate inoculation experiments and scored at day 6 or 7 (Table 2 and Figure $1 \mathrm{j}, \mathrm{k}$ ). When the three subcomponents of the total infection score were examined in detail, it was recognised that the La-er and Col-O flowers exhibited the identical infection symptoms on the new siliques, namely tissue drying and a pale grey or brown coloration. However, large differences in the levels of infection between La-er and Col$\mathrm{O}$ were evident on the flowers and older siliques. Flower
Table 1 Description of the disease phenotypes used to quantify the three subcomponents of Fusarium infection of floral tissue on Arabidopsis plants

\begin{tabular}{|c|c|c|}
\hline Organ & Score & Description of disease phenotypes \\
\hline \multirow[t]{4}{*}{ Flower (F) } & 0 & Normal \\
\hline & 1 & Aerial mycelium visible on flower \\
\hline & 3 & Drying of flowers \\
\hline & 5 & $\begin{array}{l}\text { Stem constriction within flower } \\
\text { head }\end{array}$ \\
\hline New siliques ${ }^{\mathrm{a}}$ (NS) & 0 & Normal \\
\hline \multirow{4}{*}{ Older siliques $^{b}$ (OS) } & 1 & Aerial mycelium on silique surface \\
\hline & 3 & Drying of silique surface \\
\hline & 5 & $\begin{array}{l}\text { Peduncle constriction or } \\
\text { mycelium on peduncle or loss of } \\
\text { siliques by disease travelling } \\
\text { down stem }\end{array}$ \\
\hline & 7 & Main stem constriction \\
\hline
\end{tabular}

The final FAD value is calculated by addition of the three subcomponent scores.

${ }^{a}$ Siliques formed during the disease time course.

${ }^{b}$ Siliques already formed at the time of inoculation. Both silique types are scored separately using the same scoring system.

infections in La-er were always maximum. All flowers were covered with abundant aerial mycelium, the flower petals and sepals had dried and taken on a grey or brown coloration, the supporting peduncle had also dried and constricted and these phenotypes extended into the adjacent stem tissue (Figure 1j). In comparison, the Col-O flowers showed various infection phenotypes over the 11 experiments. These ranged from only aerial mycelium on flowers (two experiments), drying and discoloration of flowers and sepals (five experiments) (Figure 1k), to all of the previous in association with some stem constriction (four experiments). This is reflected in the very high standard deviation $( \pm 1.3)$ for the flower score of 3.0 on Col-0, whereas for the La-er plants in the same 11 experiments, there was a uniform response (Table 2). For the older silique infections on La-er, these were comparable with those of the newly formed siliques, and caused the siliques to dry and display a pale grey or brown colour (Figure 1j). In contrast, the older Col-O siliques only ever exhibited superficial aerial mycelium (Figure 1k). Inoculations using another isolate of F. culmorum and an isolate of $F$. graminearum confirmed that the infections on La-er were always greater than Col-O (data not shown).

\footnotetext{
Figure 1. continued

(k) Arabidopsis ecotype Columbia (Col-O), 7 days after inoculation with F.culmorum conidia. Some aerial mycelium is associated with the flower petals and disease symptoms are present on the immature siliques.

$(\mathrm{I}, \mathrm{m})$ Tobacco flowers 4 days after inoculation with F. graminearum. (I) an emasulated tobacco flower with brown discoloration of petals but no aerial mycelium; $(\mathrm{m}$, left) a de-pistillated flower exhibiting extensive aerial hyphal colonisation of anthers and brown discoloration of petals and ( $\mathrm{m}$, right) a control non-inoculated tobacco flower at the identical physiological age to the inoculated flowers shown in (I) and $(\mathrm{m})$.

Bars $=2 \mathrm{~mm}$ in (a, g); $4 \mathrm{~mm}$ in (b, j-m); $1 \mathrm{~cm}$ in (c); $100 \mu \mathrm{m}$ in (e); $10 \mu \mathrm{m}$ in (f); $500 \mu \mathrm{m}$ in (h); $200 \mu \mathrm{m}$ in (i).
}

(c) Blackwell Publishing Ltd, The Plant Journal, (2002), 32, 961-973 
Table 2 Component scores for Fusarium culmorum floral infections of Columbia (Col-O) and Landsberg erecta (La-er) Arabidopsis plants

\begin{tabular}{|c|c|c|c|c|c|c|c|c|c|c|c|c|c|c|}
\hline \multirow[b]{2}{*}{ Genotype } & \multirow[b]{2}{*}{ Organ } & \multicolumn{11}{|c|}{ Experimental replica } & \multirow[b]{2}{*}{ Mean } & \multirow[b]{2}{*}{ SD } \\
\hline & & 1 & 2 & 3 & 4 & 5 & 6 & 7 & 8 & 9 & 10 & 11 & & \\
\hline \multirow[t]{5}{*}{ Col-O } & Flower & 2.4 & 4.4 & 4 & 0.9 & 3 & 3.2 & 0.9 & 2.3 & 4.1 & 4.8 & 2.8 & 3 & 1.3 \\
\hline & New silique & 3 & 3 & 3 & 3 & 3 & 3 & 3 & 2.8 & 3 & 3 & 3 & 3 & 0.06 \\
\hline & Older silique & 0 & 1.2 & 1.2 & 1.2 & 0 & 0 & 1.1 & 0.6 & 0.8 & 0.8 & 0 & 0.6 & 0.53 \\
\hline & Total FAD & 5.4 & 8.6 & 8.2 & 5.1 & 6 & 6.2 & 4.9 & 5.7 & 7.9 & 8.5 & 5.8 & 6.6 & 1.4 \\
\hline & & * & ** & $* *$ & * & $*$ & * & $*$ & * & $* *$ & ** & * & & \\
\hline \multirow[t]{4}{*}{ La-er } & Flower & 5 & 5 & 5 & 5 & 5 & 5 & 5 & 5 & 5 & 5 & 5 & 5 & 0 \\
\hline & New silique & 3 & 3 & 3 & 3 & 3 & 3 & 3 & 3 & 3 & 3 & 3 & 3 & 0 \\
\hline & Older silique & 2.7 & 1.9 & 2.7 & 2.1 & 3.7 & 3.6 & 1.8 & 2.4 & 3 & 3.2 & 2.6 & 2.7 & 0.64 \\
\hline & Total FAD & 10.7 & 9.9 & 10.7 & 10.1 & 11.7 & 11.6 & 9.8 & 10.4 & 11 & 11.2 & 10.6 & 10.7 & 0.64 \\
\hline
\end{tabular}

${ }^{*} \mathrm{Col}-\mathrm{O}$ single experimental mean statistically below the overall experimental FAD mean.

${ }^{* *} \mathrm{Col}-\mathrm{O}$ single experimental mean statistically above $2 \mathrm{SD}$ from the overall mean of $6.6(P<0.5)$.

The inconsistent infection phenotype of the Col-O flowers to Fusarium is curious and was observed over a 1.5-year period. Despite many modifications to the inoculation protocol and changes in the fungal isolate used, stability of both the $\mathrm{F}$ and $\mathrm{OS}$ scores was never obtained. The variation evident in the older siliques scores of Col-O was very similar to that observed in the La-er older siliques, with $S D$ values of 0.53 and 0.64 , respectively. The large variation for the Col-O flower scores appears to have two main underlying causes. Firstly, open flowers at the time of inoculation, when infected, either become pollinated and are therefore scored as new siliques, or they remain as nonpollinated flowers attached to the flower peduncle by the aerial mycelium (Figure 1k). Secondly, the closed Col-O flower buds at the time of inoculation either open and exhibit disease symptoms, open and escape infection or remain closed and disease-free. Due to the different morphology of the La-er floral apex (discussed below), neither uninfected open flowers or uninfected closed buds were observed (Figure 1j).

When the combined data in Table 2 was explored in greater detail, an excellent positive correlation was evident between the overall level of floral disease in a single experiment (i.e. the FAD value) and the extent of disease symptoms on the flowers $(r=0.93)$. However, there was only a poor positive correlation between the total FAD value and the disease score of the older siliques $(r=0.38)$. No correlation was found between the disease levels on the flowers and older siliques $(r=0.01)$. Therefore, the limited infection levels on the older Col-O siliques are not caused from an overall lower infection level in a single experiment or from less fungal biomass on the flowers. In the same experimental series, the three subcomponents of the Laer FAD values remained consistent (Table 2). Overall, the total $F A D$ values for $\mathrm{Col}-\mathrm{O}$ was bimodal in distribution, with seven experiments giving mean FAD scores statistically below the overall mean and four experiments giving values statistically above the mean (Table 2). For La-er, a normal distribution was obtained, with the only difference between experiments caused by the degree of colonisation of the older siliques.

The most plausible explanation for the higher infection phenotype of La-er compared to Col-O flower heads is the large difference in their flower head architecture. The La-er flower head is extremely compact, with immature siliques, open flowers and unopened buds in extremely close proximity to each other at the time of inoculation (Figure 1j). In comparison, the Col-O flower head is less compact with each open flower slightly more separated from the unopened flower buds. Also, a space of at least 4-6 $\mathrm{mm}$ separates an open flower from immature siliques at the time of inoculation (Figure 1k). These architectural differences will greatly affect the propensity of the Fusarium aerial mycelium formed at the initial site of flower infection to grow aerially into adjacent siliques and other flowers, and for the various infection sites to coalesce. The maximal aerial range of these Fusarium hyphae is around $2 \mathrm{~mm}$ for Arabidopsis, although considerable greater aerial extension was evident on wheat and tobacco. In addition, it is highly likely that the overall humidity within the tight La-er flower head could remain consistently higher than that in the more lax Col-O flower heads, even though high-humidity chambers were used throughout the experimental time course. Fusarium infections always proceed faster under very high humidity conditions because of the greater contribution of the aerial mycelium to initiate new infection sites (Martin Urban, unpublished data). We consider that the higher severity of infection on the older La-er siliques already formed at the time of infection could be the result of underlying genetic differences in the two ecotypes' abilities to respond to the Fusarium infection. However, because of the compact La-er flower head, the vertical growth of the older siliques causes them to come into contact with the abundant aerial mycelium. A high proportion of secondary 
infections then take place, which could also elevate the overall disease score. Wheat cultivars with a compact and dense ear architecture are known to be generally more susceptible to Fusarium ear blight infection than those with loose and open ear morphologies (reviewed by Parry et al., 1995).

Screening a diverse collection of Arabidopsis ecotype for genetic variation in both flower and leaf infection phenotypes

In total, 236 Arabidopsis ecotype accessions were spray inoculated with Fusarium when the primary bolt of each plant had at least two open flowers and no more than two siliques had already formed. A minimum of five inoculated plants per ecotype were assessed for visible flower, silique, stem and leaf infections, at either 7 or 8 days post-inoculation. Twenty-five ecotypes in the primary screen with FAD values below 3 or above 10 were re-grown and inoculated, with the population size increased to 12 flowering plants. None of these extreme FAD values re-confirmed during the secondary screen. We therefore conclude that all 236 Arabidiposis ecotypes are moderately susceptible to floral head infections. No ecotype exhibited visible leaf infection symptoms (http://www.iacr.ac.uk/ppi/staff/khkara.html).

The extent of Fusarium floral infections is significantly affected by the presence and absence of functional anther components

A key route for Fusarium to invade floral tissue involves the anthers. In wheat, when the anthers are carefully removed by physical emasculation, then the incidence of infections can be dramatically reduced (Strange and Smith, 1971). Two metabolites highly stimulatory to Fusarium hyphal growth and mycelium branching are found in abundance in anthers and pollen. Choline and glycine betaine (Strange et al., 1974) have been suggested to be positive contributors to fungal pathogenicity and possibly explain why F.graminearum and F. culmorum invade flowers. Maximum cereal ear invasion always occurs at anthesis. We therefore wanted to explore whether there was a functional significance to the anther invasions observed on the four dicotyledonous species.

The large size of the tobacco flowers meant this plant species was the most experimentally tractable of the four to examine the effects of hand-emasculation and de-pistillation on the Fusarium-infection phenotype. When all the pollen sacs were removed from the ends of the anther filaments of tobacco flowers prior to pollen dehiscence, very little aerial Fusarium mycelium was evident on the remaining filament tissue post-inoculation and the petals turned brown (Figure 11). Microscopic analyses revealed internal colonisation of petal tissue (data not shown). In contrast, when the pistil was removed prior to anther dehiscence and inoculation, the heavily infected flower remained alive for a few additional days, but no colonisation of the underlying pistil and unfertilised ovary and sepal tissues occurred (Figure 1m).

In Arabidopsis, a wide range of flower mutants exist that have either the entire floral whorls missing or present in duplicate, whilst other mutations result in no silique formation (http://nasc.nott.ac.uk; http://arabidopsis.org). We decided to examine the effects of the various genetic ablations of anther whorls' development or pollen function on the FAD values in preference to hand emasculation. Three floral mutants were selected for these experiments: agamous- 1 because it lacks the pistal and stamen whorls and instead possesses a double petal whorl (Yanofsky et al., 1990); apetala3-1 because it lacks both the stamen and petal whorls, and has instead double carpel and sepal whorls (Jack et al., 1992). The ag-1 and ap3-1 mutants are both in the La-er ecotype backgrounds. The third mutant was the recently reported jasmonic acid mutant called dad1, present in the Ws-2 background (Ishiguro et al., 2001). The dad1 mutant has all the floral whorls intact, but has defective pollen dehiscence and filament hydration processes and the pollen is non-viable. In all three mutants, pollination does not occur. Instead residual, undeveloped ovules and pericarp tissue in a silique-like structure remain at the end of the flower peduncle following final petal and/or sepal senescence.

Interestingly, all three mutants showed significantly reduced Fusarium infection of flowers. For the agamous1 mutant, very little aerial mycelium formed and the flowers appeared normal. The individual flower infection score was La-er $=5$ and $a g-1=1$. Sometimes, slow petal senescence occurred in the agamous-1 mutant under the high-humidity conditions and this caused saprophytic aerial mycelium to become visible only on the petals from day 5 onwards. However, this petal phenotype did not result in additional colonisation of other parts of the flower head. On the apetala3-1 mutant, some aerial white mycelium was evident on the undeveloped ovary and calyx whorls by day 5 . But, again no further disease symptoms or tissue browning ever occurred. The inoculation score of the flower phenotype for ap3-1=1.0. We therefore conclude that the complete ablation of the anther tissue whorl prevents Fusarium hyphae from invading other tissues within the flower head.

The dad1 mutant also exhibited a very low disease phenotype, with no macroscopically visible hyphae on flowers and minimal aerial hyphae on the surface of the non-fertilised siliques. The F+NS score for the wild-type Ws-2 ecotype $=4.2$, whereas for $\mathrm{dad} 1=2.1$. This result was not expected because the anther whorl is present, although non-functional. When inoculated dad1 and wild-type Ws-2 flowers were examined microscopically over a 2-day time course extending to day 10 , this revealed that almost no 
extracellularly mycelial colonisation of the anther and anther filament has occurred on the dad 1 flowers and there was no evidence of attempted tissue invasion. In the Ws-2 flowers, extracellularly mycelial colonisation was extensive and other intercellular and intracellular hyphae were detected in the peduncle and silique tissues (data not shown). It would therefore appear that Fusarium hyphae lack the appropriate mechanism to penetrate the intact anther sac wall and do not use, as an alternative route, the 4-6 stomatal pores present near the junction with the anther filament. Also, the overall reduction in the density of hyphae associated with dad1 anthers compared with Ws-2 may indicate a lower nutrient availability. Alternatively, it is possible that the intact anthers containing immature nonviable pollen grains either contain harmful pre-formed metabolites (phytoanticipins) (Morrissey and Osbourn, 1999) or are able to mount a successful active defence response at sites of attempted hyphal penetration. Microscopic analyses revealed no evidence of a hypersensitive cell death defence response or any specific modifications to the appearance of plant cell walls in either the anther sac or filament (data not shown).

The small amount of belated aerial mycelia evident on all three of the Arabidopsis flower mutants on the unfertilised and senescing calyx, siliques and petals (where present) never resulted in disease symptoms forming on the underlying peduncle or stem tissues. The formation of aerial mycelium on these tissues was to be expected because Fusarium has excellent saprophytic colonisation abilities, and can therefore utilise the considerable nutrients released from these tissues as each enters a senescence programme.

\section{DON mycotoxin production during invasion of Arabidopsis flowers}

A feature of many F.graminearum and F.culmorum invasions of wheat and barley ears and maize cobs is the production of mycotoxins (Hohn et al., 1998). The trichothecene mycotoxin, deoxynivalenol (DON) and its precursor 15-acetyl deoxynivalenol are of concern for human food and animal feed safety (http://www.scabusa.org). The production of DON mycotoxins may contribute to the overall aggressiveness of naturally DON-producing Fusarium isolates to invade plant tissues. However, DON production is not a fundamental fungal pathogenicity factor (diseasecausing component) (Harris et al., 1999; Proctor et al., 1995). DON production can also be induced under specific in vitro conditions that attempt to mimic in planta conditions, but DON and 15-acetyl is not synthesised when F. culmorum or F. graminearum are grown on more general culture media (Burmeister, 1971; Chen et al., 2000).

We explored whether DON and 15-acetyl DON production occurred during Fusarium colonisation of Arabidopsis floral tissue. Samples were taken at 8 days post-inoculation with two different F.culmorum isolates and one F.graminearum isolate known to produce DON mycotoxin when invading wheat ears (Claudia Heppner, unpublished). In both Col-O and La-er whole flowers, greater than $1 \mathrm{ppm}$ DON was detected in all Fusarium-invaded flower tissue at 8 days post-inoculation. On Col-O flowers, the recovered values ranged between 1.22 and $1.42 \mathrm{ppm}(S D=0.10)$ whilst on La-er, a slightly wide range was evident from 1.21 to $2.27 \mathrm{ppm}$ (SD=0.53). From water only-inoculated Col-O and La-er floral tissue, DON levels of below 0.1 ppm were recorded. This level of background non-specific binding of plant components in the DON competitive ELISA is comparable to the values obtained for water only-inoculated Bobwhite wheat ears (C. Heppner, unpublished). When the two UK F. culmorum isolates were spray inoculated onto the anthesising ears of Bobwhite wheat growing in a field in Cambridgeshire, UK and subsequently mist irrigated to ensure a high level of visible infection, the DON levels recovered from the harvested and threshed grain ranged between 6 and $50 \mathrm{ppm}$ (C. Heppner, unpublished). This indicated that the two F. culmorum isolates used in this study are moderate producers of DON. The large difference in the DON levels recovered from the wheat and Arabidopsis inoculation would appear to be caused by the total length of the infection period prior to assaying for DON levels. Only 8 days elapsed between inoculation and floral tissue harvest in Arabidopsis, whereas there are 50 days between inoculation and harvest for the wheat grain sample. In the later situation, considerably more time elapsed for mycotoxin biosynthesis and accumulation to occur.

The detection of the accumulation of DON and 15-acetyl DON mycotoxins in the Fusarium-infected flowers of both Col-O and La-er flowers indicates that the complete infection phenotype is manifested within Arabidopsis floral tissue.

\section{Discussion}

F. graminearum and F. culmorum are primarily considered to be pathogens of monocotyledonous cereal plant species and cause the serious ear blight disease (McMullen et al., 1997). The combined data presented in this analysis clearly indicates that both fungal species are able to penetrate and invade the floral tissue of various dicotyledonous species and for Arabidopsis, these infections extend into stem tissue to cause severe disease symptoms, and the accumulation of DON mycotoxins and hyphae to associate with developing seeds inside infected siliques. This is a novel pathosystem for Arabidopsis and is the first to be described involving floral infection by any microbe. The lack of leaf tissue infections also highlights a frequently overlooked aspect of plant-microbe interactions, the phenomenon of organ specificity and/or tissue specificity for successful 
microbial colonisation. Preliminary results using another wheat floral pathogen Stagnospora (Septoria) nodorum also indicates that this fungal species is able to invade and cause visible disease on tobacco flowers and Arabidopsis flowers, siliques and upper stem tissues (Martin Urban, personal communication).

\section{Mode of infection}

The mode of infection by Fusarium hyphae of the four dicotyledonous plant species, i.e. Arabidopsis, tobacco, tomato and soybean was very similar to that found in wheat and barley. Under high-humidity conditions, floral tissue was invaded but not leaf or lower stem tissues. The microscopic observations of the trypan blue-lactophenol-stained tissue indicate that the advancing hyphal front colonised living plant tissue, and therefore the pathogen is hemibiotrophic in these dicotyledonous plant species. Why F.graminearum and F.culmorum are primarily flower and stem base invaders is not known. This may be because of: (i) the local abundance of specific metabolites within the flowers, e.g. choline and glycine betaine; (ii) the excessive nutrients available post-fertilisation in the primarily sink tissues or (iii) tissues with no or highly reduced photosynthetic capacity have a different baseline primary metabolism. The extensive aerial mycelia that developed in association with anther tissue early in the infection was a striking feature of all five pathosystems. However, only when attacking the flowers of monocotyledonous cereal plants and Arabidopsis did the Fusarium infections spread into other floral tissues to eventually reach the stem and developing seeds. In tobacco, tomato and soybean, the infections were always contained within the open flowers and these infections were excised completely at flower senescence. It is currently unclear why these infections fail to spread further.

Within Arabidopsis floral tissue, both extensive intercellular and intracellular invasion occurred and this led to tissue drying and browning in association with asexual spore production. The same disease symptoms are seen in wheat and barley ears. However, unlike in wheat, the Arabidopsis infections invariably resulted in the diseased floral heads necking over at the point where the stem tissue became invaded, late in the time course. Infected wheat and barley ears remain vertical and firm even under periods of extended high humidity. We consider this major difference in disease symptoms to be primarily due to differences in the physiological age of the floral tissue invaded by the fungal hyphae and hence the degree of secondary cell wall modifications, i.e., lignification, that has occurred. At anthesis, the entire floral structure in both wheat and barley ear is approximately 30 days old. Whereas for Arabidopsis, at the onset of anthesis, the floral head is $<10$ days old and is considerably more supple.
The Arabidopsis floral mutants, agamous-1, apetala3-1 and dad 1 confirm the need for the presence of both anthers and pollen function to permit the successful and extensive invasion of other floral tissues. An analysis of various male sterile barley genotypes (Matsui et al., 2002) has revealed that lines producing sterile pollen were significantly more susceptible to Fusarium ear blight than sterile lines producing no pollen. The negative infection results with the Arabidopsis dad 1 mutant suggests that Fusarium may have difficulties penetrating non-dehiscing anther sacs. The emasculated tobacco flower results reveal that petal infections can take place even in the absence of pollen in this species. In contrast, another cereal floral fungal pathogen Claviceps purpurea, the causal agent of ergot disease, only enters floral tissue via the female stigmatic tissues (Mey et al., 2002).

\section{Resistance}

Both F. graminearum and F.culmorum lack the ability to invade unwounded plant leaf tissue of both monocotyledonous and dicotyledonous plant species. No infection occurs even in the presence of detached anthers. Instead, infections by these two Fusarium species are confined to either the flowers or the stem bases. This tissue- or organspecific resistance suggests one of three potential hypotheses: (i) specific signals are missing to trigger the onset of the Fusarium infection process; (ii) specific defence responses operate in the non-infected organs prior to, or during attempted penetration or (iii) in the predominantly non-photosynthetically active plant tissue, a full defence response cannot be manifested and this permits the fungal hyphae to gain entry. In wheat, F. graminearum infection of floral tissue triggers the induction of the typical set of defence-related genes (Pritsch et al., 2001). Amongst the Fusarium genera, it is noticeable that most species occupy just specific tissue niches within the plant and do not attack every plant organ. For example, Fusarium wilt fungi such as F. oxysporum $\mathrm{fsp}$. lycopersici are restricted to attacking the vascular tissue near the stem base (Agrios, 1997), whilst F. oxysporum f. sp. matthiolae, the only other Fusarium species reported to attack Arabidopsis, is primarily a stem base-root coloniser (Epple et al., 1998).

The Arabidopsis ecotype screen had a disappointing outcome, with no extremely resistant or susceptible ecotypes identified. A similar outcome was found when a large number of Arabidopsis ecotyopes were inoculated with the economically important sugar beet cyst nematode species Heterodera schactii. Although statistically significant differences between La-er and Col-O infection phenotypes were repeatedly evident, these differences have probably more to do with overall floral architecture than inherent differences in their resistance response. One of the easiest ways to test this hypothesis is to transform the Landsberg 
allele of the erecta gene into the Col-O background and repeat the Fusarium inoculation tests.

In wheat, two main types of germplasm resistance are recognised. Type 1 resistance prevents initial infection, whilst type 2 resistance operates post-infection to reduce the rate of secondary spread through the entire ear (reviewed by Parry et al., 1995). The spray inoculation protocol undertaken in this study is very useful in trying to identify type 1 and type 2 resistance components together. However, to focus specifically on type 2 resistance (i.e., resistance to spread) would require the use of a single flower point inoculation technique. In wheat, a single spikelet inoculation technique is frequently used to identify germplasm exhibiting good type 2 resistance (Schroeder and Christensen, 1963).

\section{Exploitation}

This novel Fusarium floral pathosystem on Arabidopsis will now permit a study of various defence mutants and transgenics for their ability to restrict or enhance disease susceptibility and to alter symptomatology. A wide range of defence mutants already exist for Arabidopsis unlike wheat or maize. In parallel with these inoculation experiments, it will be important to determine whether the various genetic mutations that affect the levels of specific defence-signalling molecules, for example salicylic acid, jasmonic acid, ethylene and reactive oxygen species actually cause similar changes in floral tissues as reported for leaves. For most of the mutants, this type of information has not been reported. Large-scale gene expression array experiments could also be undertaken to examine the global changes in the transcriptome caused during Fusarium infection of floral tissue. These gene expression experiments could be extended to leaf tissue to determine whether plant cells actively respond during surface colonisation by non-penetrating Fusarium mycelium.

The biological complexity of the Fusarium infection process in floral tissue and the lack of specific infection structures at the site of initial host tissue penetration, i.e. appressoria formation means that any mutation screen would best be achieved by using a transformed isolate of Fusarium expressing both the green fluorescent protein (GFP) and $\beta$-glucuronidase (GUS) reporter genes. This would permit an initial non-destructive and high-throughput analyses to be performed using UV to detect the presence of the green fluorescent protein within the fungal hyphae. This would be followed by GUS staining and light, epi-fluorescence and confocal microscopic analyses to evaluate the true extent of internal Fusarium colonisation. A recent study using a transgenic Fusarium strain constitutively expressing the GFP gene reporter was used to document Fusarium invasion of wheat ears (Pritsch et al., 2001).
The detection of DON mycotoxin production within the Fusarium-infected floral tissue will also permit a detailed examination of the plant factors regulating mycotoxin biosynthesis. This type of analysis, which could be undertaken using monocotyledonous and/or dicotyledonous host plant species, is also best achieved using a transgenic Fusarium strain because the DON ELISA and GC-MS tests are both expensive and requires large amounts of infected plant tissue $(>4 \mathrm{~g}$ ). One suitable transgenic Fusarium strain to monitor DON induction could harbour a Tri5 promoter: GFP-GUS reporter gene construct. The Tri5 gene of Fusarium encodes for the enzyme trichodiene synthase, and this is the key regulatory step for DON mycotoxin production (Proctor et al., 1995). By using a pTri5:GFP-GUS transgenic Fusarium isolate, the onset of DON biosynthesis can be pinpointed to specific infection stages and plant tissues. Then, commercial monoclonal antibodies to DON can be used on tissue sections to confirm associated DON production. By this approach, will it be possible to compare DON production in various mutant and transgenic Arabidopsis lines to that occurring in wheat and barley ears and maize cobs.

\section{Experimental procedures}

\section{Fusarium strains and media}

The two F. culmorum isolates used in this study were strains $97 / 7$ and $98 / 11$ which had been isolated from naturally infected wheat ears taken from the Trumpington Cambridge site in 1997 and 1998 respectively (Bill Hollins, personal communication). The F. graminearum isolate 16A originated from the USA, was isolated from infected wheat grain collected from a wheat grain elevator in Montana in 1997 and was a gift from Linda Lahman (Monsanto, St. Louis). All three isolates were capable of causing severe ear blight symptoms on susceptible wheat cultivars and to produce DON mycotoxins (M. Urban and C. Heppner, unpublished data).

Both F. culmorum and F. graminearum were propagated on SNA plates (synthetic nutrient poor agar) containing $0.1 \% \mathrm{KH}_{2} \mathrm{PO}_{4}, 0.1 \%$ $\mathrm{KNO}_{3}, 0.1 \% \mathrm{MgSO}_{4} .7 \mathrm{H}_{2} \mathrm{O}, 0.05 \% \mathrm{KCl}, 0.02 \%$ glucose, $0.02 \%$ saccharose, $2 \%$ Bacto agar (Difco) supplemented with 200 ppm biotin and $200 \mathrm{ppm}$ thiamine. For long-term storage in liquid nitrogen, conidiospore suspensions were prepared to a density of $10^{7}$ spores $\mathrm{ml}^{-1}$ in $10 \%$ glycerol. Spore suspensions for plant inoculations were derived from culture plates which had been subcultured a maximum of four times. Conidiospores were harvested from $90 \mathrm{~mm}$ SNA agar plates after 10 days incubation by adding $5 \mathrm{ml}$ sterile water and scraping off conidiospores with a spatula. Alternatively bubble cultures (Cappellini and Peterson, 1965) were established to create a greater volume of conidiospores. The spores were recovered by centrifuging at $3000 \mathrm{~g}$ for $6 \mathrm{~min}$ at $4^{\circ} \mathrm{C}$, then re-suspended gently in growth media and stored for up to 7 days at $4^{\circ} \mathrm{C}$ prior to use. For plant inoculations, aliquots of spores were taken from the stock, recovered by centrifuging at $3000 \mathrm{~g}$ for $6 \mathrm{~min}$ at $4^{\circ} \mathrm{C}$, washed once with sterile de-ionised water, centrifuged again and re-suspended in water plus $0.001 \%$ Silwet $\mathrm{L}$ 77 to give a final spore concentration of $1 \times 10^{5}$ spores $\mathrm{ml}^{-1}$. Conidiospores were counted using a haemocytometer. All experiments 
involving the F.graminearum isolate $16 \mathrm{~A}$ were conducted in biological containment facilities under DEFRA licences PHL 39B/3819 (5/2001) and PHL 39A/3493 (01/2001).

\section{Growth of plant material}

Arabidopsis seed was sown into Levingtons F1 compost and kept in a propagator for 4 days at $4^{\circ} \mathrm{C}$ to ensure even germination. The pots of germinated seeds were then moved to a controlled environment growth room with a temperature of $20^{\circ} \mathrm{C}$ and a 12-h light/ 12-h dark cycle. Light was supplied by a mixture of metal halide and incandescent lamps to produce a fluence level of 171 microM at $64.5 \mathrm{~W} \mathrm{msq}^{-1}$ at the plant surface. Ten days later, at the one leaf stage, seedlings were pricked into vacupots (24 units per tray), again into F1 compost. Plant pots were placed on capillary matting, which was kept moist at all times. Flowering commenced approximately 3 weeks after the seedling were pricking out. Under these environmental conditions, the full ap3-1 Arabidopsis mutant phenotype was always evident.

Tobacco seeds of cultivar Petit Havana, tomato seeds of cultivar Moneymaker (Cf-0) and soybean seeds of cultivar A3244 were sown in Levingtons F1 compost, and pricked out singly into Levingtons F2 compost at the 2-4 leaf stage into $10-\mathrm{cm}$ pots. The plants were grown in a controlled environment growth room at $24^{\circ} \mathrm{C}$ during the $16-\mathrm{h}$ day and $16^{\circ} \mathrm{C}$ at night. Light was supplied by a mixture of metal halide and incandescent lamps to produce a fluence level of 207 microM at $86.2 \mathrm{~W} \mathrm{msq}^{-1}$ at the plant surface.

Wheat seeds of spring cultivar Bobwhite were sown in Levingtons C2 coarse potting compost for 2 months in a controlled environment growth room at $18^{\circ} \mathrm{C}$ during the 16 -h day and $16^{\circ} \mathrm{C}$ during the 8 -h night at $50 \%$ relative humidity. Light was supplied by a mixture of metal halide and incandescent lamps to produce a fluence level of 207 microM at $86.2 \mathrm{~W} \mathrm{msq}^{-1}$ at the plant surface.

\section{Plant inoculations}

Only flowering plants were selected for inoculation. The Arabidopsis plants possessed an unbranched bolt with both open flowers on the terminal inflorescence and two to three developing siliques. Ten plants from each genotype were selected for each Fusarium inoculation, and a further two to three control plants to be sprayed with water. A permanent black marker pen was used to mark the position on the flower stem above which only open flowers were present and below which siliques had already set. Tobacco, tomato, soybean and wheat plants were selected just as flowering/anthesis commenced. Six plants of each species were selected for each experiment. One to three flowers per plant were inoculated. A randomised block experimental design was used to minimise experimental error and each experiment was conducted a minimum of three times.

For the inoculation of Arabidopsis flowers, a fine droplet spray applicator was used. The spore suspension was applied until droplet run-off had just commenced. After this, each inflorescence was re-inoculated with the inoculum dispensed this time from a medical nasal applicator, four puffs per flower head. Control plants were inoculated in the same way using de-ionised water. The inoculated plants were then kept in large plastic propagators at $100 \%$ relative humidity for the next 7 days. For the first 2 days, the chambers were shaded with capillary matting to exclude light. A similar dark procedure is used when inoculating wheat ears. Individual plants were scored for disease symptoms from day 2 onwards with the final disease score taken at day 7 post-inoculation.
For the inoculation of the tobacco flowers, soybean flowers and wheat ears, the inoculum was dispensed from the medical nasal applicator, four puffs per open flower and eight puffs per wheat ear. The anther cones of the tomato flowers are extremely waxy and this caused most spore droplets to be lost immediately through run-off. Therefore, a single segment of the tomato flower anther cone was removed by the use of a pair of fine forceps. Then, using a P10 Gilson pipette, $5 \mu \mathrm{l}$ of spore suspension was added to the inside of each anther cone. After inoculation, all the plants were treated as described above for the Arabidopsis inoculations.

\section{Disease scoring on Arabidopsis}

To quantify accurately the levels of disease symptoms visible on the various Arabidopsis floral tissues, a numerical scoring system was devised. This is shown in Table 1. The disease phenotypes were assessed for three separate floral subcomponents, namely flowers $(F)$ that were either open flowers or closed buds at inoculation, new siliques (NS) that were fully open flowers at inoculation (i.e. located above the permanent mark placed on the stem) and older siliques (OS) already present at inoculation. An increasing numerical score was used to quantify the abundance of aerial mycelium on a tissue surface $(0,1)$, as well as the increasing severity of the disease symptoms visible on plant tissue as the invasion process progresses (3-7). The intermediate scores of 2 and 4 (F), and 2, 4 and 6 (NS and OS) were reserved for when all the tissue on a single plant exhibited the disease phenotype described for the preceding score. For example, $100 \%$ drying of flowers received a score of 4 . The final Fusarium-Arabidopsis disease (FAD) value was calculated by addition of the three subcomponent scores, i.e. $\mathrm{F}+\mathrm{NS}+\mathrm{OS}=\mathrm{FAD}$. Arabidopsis genotypes with $\mathrm{FAD}$ values of 3 and below were classified as exhibiting resistance to Fusarium ear blight whereas those with values of 10 and above were classified as susceptible.

\section{Plant infection studies}

Floral tissue was taken at 2, 3, 4, 5, 6 and 7 days after inoculation from each genotype/Fusarium isolate interaction. Conventional histochemical staining of fungal hyphae was performed using lactophenol-trypan blue and de-staining with chloral hydrate (Keogh et al., 1980). Microscopic observations were made on a Carl Zeiss 'Axioskop 2' instrument under phase contrast and photomicrographs were prepared with Kodak Ektachrome 160 Tungsten films. Statistical analyses were conducted according to Snedecor and Cochran (1980) and using either the SAS statistical package or the data analysis tools in MS Excel.

\section{DON determinations}

Four grams of infected Arabidopsis floral tissue from La-er and Col-O plants was harvested 8 days post-Fusarium-inoculation, frozen in liquid nitrogen and stored at $-80^{\circ} \mathrm{C}$. Similar samples were collected from water-inoculated control plants. The frozen tissue samples were ground to a fine powder in a mortar using a pestle in the presence of liquid nitrogen. One gram of each sample was then re-suspended in $3 \mathrm{ml}$ of water and thoroughly mixed using a polytron for $30 \mathrm{sec}$. The mixture was then incubated for $30 \mathrm{~min}$ at $30^{\circ} \mathrm{C}$ in a water bath. All solid parts were removed by centrifugation and the supernatants were analysed for mycotoxin content. Quantitative analysis of combined DON and its precursor 15-acetyl DON measurements were made using the commercially approved 
competitive ELISA Veratox 5/5 kit (Adgen, Ayr, Scotland) and deploying a standard curve for DON ranging from 0.25 to $3.00 \mathrm{ppm}$. OD650 values were measured $5 \mathrm{~min}$ after the addition of the stop solution to the multiwells. To ensure accuracy, each biological same was quantified twice for combined DON and 15acetyl DON levels.

\section{Acknowledgements}

We wish to thank Dee Shelford for assistance with plant care and seed harvest and Dr Brian Church for some of the statistical analyses. This research was supported by Monsanto.

\section{References}

Agrios, G.N. (1997) Plant Pathology, 4th edn. San Diego, CA, USA: Academic Press Inc.

Anderson, J.A., Stack, R.W., Lui, S. et al. (2001) DNA markers for Fusarium head blight resistance QTLs in two wheat populations. Theor. Appl. Genet. 102, 1164-1168.

Ban, T. and Suenaga, K. (2000) Genetic analysis of resistance to Fusarium head blight caused by Fusarium graminearum in Chinese wheat cultivar Sumai 3 and the Japanese cultivar Saikai 165. Euphytica, 113, 87-99.

Buerstmayr, H., Lemmens, M., Grausgruber, H. and Ruckenbauer, P. (1997) Breeding for scab resistance in wheat: inheritance of resistance and possibilities for in vitro selection. In Fusarium Head Scab: Global Status and Future Prospects (Dubin, H.J., Gilchrist, L., Reeves, J. and McNab, A., eds). Mexico: CIMMYT, pp. 52-58.

Burmeister, H.R. (1971) T-2 toxin production by Fusarium tricinctum on solid substrate. Appl. Microbiol. 21, 739-742.

Cappellini, R.A. and Peterson, J.L. (1965) Macroconidium formation in submerged cultures by a non-sporulating strain of Gibberella zeae. Mycologia, 57, 962-966.

Chen, L.F., McCormick, S.P. and Hohn, T.M. (2000) Altered regulation of 15-acetyldeoxynivalenol production in Fusarium graminearum. App. Environ. Microbiol. 66, 2062-2065.

Crozier, J. and Boothroyd, C.W. (1959) Tomato: a new suscept of Gibberella zeae (Schw). Plant Dis. Reporter, 43, 446-447.

Di Pietro, A., Garcia-Maceira, F.I., Meglecz, E. and Roncero, M.I.G. (2001) A MAP kinase of the vascular wilt fungus Fusarium oxysporum is essential for root penetration and pathogenesis. Mol. Microbiol. 39, 1140-1152.

Epple, P., Vignutelli, A., Apel, K. and Bohlmann, H. (1998) Differential induction of the Arabidopsis thaliana Thi2.1 gene by Fusarium oxysporum f. sp. matthiolae. Mol. Plant-Microbe Interact. 11, 523-529.

Gang, G., Miedaner, T., Schuhmacher, U., Schollenberger, M. and Geiger, H.H. (1998) Deoxynivalenol and nivalenol production by Fusarium culmorum isolates differing in aggressiveness towards winter rye. Phytopathology, 88, 879-884.

Harris, L.J., Desjardins, A.E., Plattner, R.D., Nicholson, P., Butler, G., Young, J.C., Weston, G., Proctor, R.H. and Hohn, T.M. (1999) Possible roles of trichothecene mycotoxins in virulence of Fusarium graminearum on maize. Plant Dis. 83, 954-960.

Hohn, T.M., Krishna, R. and Proctor, R.H. (1999) Characterization of a transcriptional activator controlling trichothecene toxin biosynthesis. Fungal Genet. Biol. 26, 224-235.

Hohn, T.M., McCormick, S.P., Alexander, N.J., Desjardins, A.E. and Proctor, R.H. (1998) Function and biosynthesis of trichothecenes produced by Fusarium species. Mol. Genet. Host-Specific Toxins Plant Dis. 13, 17-24.
Hui, Y., Evans, C.K., Kolaczkowski, E.K., Dill-Macky, R. and Mirocha, C.J. (1997) Chemistry, physiology and role of deoxynivalenol in pathogenicity. Bull. Inst Compr. Agr. Sci. Kinki Univ. 5, 1-11.

Ishiguro, S., Kawai-Oda, A., Ueda, J., Nishida, I. and Okada, K. (2001) The DEFECTIVE IN ANTHER DEHISCENCE1 gene encodes a novel phospholipase $A 1$ catalyzing the initial step of jasmonic acid biosynthesis, which synchronizes pollen maturation, anther dehiscence, and flower opening in Arabidopsis. Plant Cell, 13, 2191-2209.

Jack, T., Brockman, L.L. and Meyerowitz, E.M. (1992) The homoeotic gene APETALA3 of Arabidopsis thaliana encodes a MADS box and is expressed in petals and stamens. Cell, 68, 683-697.

Jenkinson, P. and Parry, D.W. (1994) Isolation of Fusarium species from common broad-leaved weeds and their pathogenicity to winter wheat. Mycol. Res. 7, 776-780.

Kang, Z. and Buchenauer, H. (1999) Immunocytochemical localisation of Fusarium toxins in infected wheat spikes by Fusarium culmorum. Physiol. Mol. Plant Pathol. 55, 275-288.

Kang, Z. and Buchenauer, H. (2000) Ultrastructural and immunocytochemical investigations of pathogen development and host responses in resistant and susceptible wheat spikes infected by Fusarium culmorum. Physiol. Mol. Plant Pathol. 57, 255-268.

Keogh, R.C., Deverall, B.J. and Mcleod, S. (1980) Comparison of histological and physiological responses to Phakopsora pachyrhizi in resistant and susceptible soybean. Trans. Br. Mycol. Soc. 74, 329-333.

Matsui, K., Yoshida, M., Ban, T., Komatsuda, T. and Kawada, N. (2002) Role of male sterile cytoplasm in resistance to barley yellow mosaic virus and Fusarium head blight in barley. Plant Breeding, 121, 237-240.

McCormick, S.P., Hohn, T.M., Desjardins, A.E., Proctor, R.H. and Alexander, N.J. (1998) Role of toxins in plant microbial interactions. Phytochem. Signals Plant-Microbe Interact. 32, 17-30.

McMullen, M., Jones, R. and Gallenberg, D. (1997) Scab of wheat and barley: a re-emerging disease of devastating impact. Plant Dis. 81, 1340-1348.

Mey, G., Oeser, B., Lebrun, M.H. and Tudzynski, P. (2002) The biotrophic, non-appressorium forming grass pathogen $C$. purpurea needs a Fus $3 / P m k 1$ homologous MAP kinase for colonization of rye ovarian tissue. Mol. Plant-Microbe Interact. 15, 303-312.

Miedaner, T., Reinbrecht, C. and Schilling, A.G. (2000) Association among aggressiveness, fungal colonization, and mycotoxin production of 26 isolates of Fusarium graminearum in winter rye head blight. J. Plant Dis. Protect. 107, 124-134.

Morrissey, J.P. and Osbourn, A.E. (1999) Fungal resistance to plant antibiotics as a mechanism of pathogenesis. Microbiol. Mol Biol. Rev. 63, 708-724.

Nelson, P.E., Desjardins, A.E. and Plattner, R.D. (1993) Fumonisins, mycotoxins produced by Fusarium species: biology, chemistry, and significance. Annu. Rev. Phytopathol. 31, 233-252.

Parry, D.W., Jenkinson, P. and McLeod, L. (1995) Fusarium ear blight (scab) in small grain cereals - a review. Plant Pathol. 44, 207-238.

Pritsch, C., Vance, C.P., Bushnell, W.R., Somers, D.A., Hohn, T.M. and Muehlbauer, G.J. (2001) Systemic expression of defence response genes in wheat spikes as a response to Fusarium graminearum infection. Physiol. Mol. Plant Pathol. 58, 1-12.

Proctor, R.H., Hohn, T.M. and McCormick, S.P. (1995) Reduced virulence of Gibberella zeae caused by disruption of a trichothecene toxin biosynthetic gene. Mol. Plant-Microbe Interact. 8 , 593-601. 
Pugh, G.W. (1933) Factors affecting infection of wheat heads by Gibberella saubinetti. J. Agr. Res. 46, 771-797.

Schroeder, H.W. and Christensen, J.J. (1963) Factors affecting the resistance of wheat to scab caused by Gibberella zeae. Phytopathol. 53, 831-838.

Snedecor, G.W. and Cochran, W.G. (1980) Statistical Methods. Ames, IA: lowa State University Press.

Strange, R.N., Majer, J.R. and Smith, H. (1974) The isolation and identification of choline and betaine as the two major components in anthers and wheat germ that stimulate Fusarium graminearum in vitro. Physiol. Plant Pathol. 4, 277-290.

Strange, R.N. and Smith, H. (1971) A fungal growth stimulant in anthers which predisposes wheat to attack by Fusarium graminearum. Physiol. Plant Pathol. 1, 141-150.

Tag, A.G., Garifullina, G.F., Peplow, A.W., Ake, C., Phillips, T.D., Hohn, T.M. and Beremand, M.N. (2001) A novel regulatory gene,
Tri10, controls trichothecene toxin production and gene expression. Appl. Environ. Microbiol. 67, 5294-5302.

Ueno, Y., Ishikawa, Y., Saito-Amakai, K. and Tsunoda, H. (1970) Environmental factors influencing the production of Fusarenon$\mathrm{X}$, a cytotoxic mycotoxin of Fusarium nivale Fn2B. Chem. Pharm. Bull. 18, 304-312.

Van Ginkel, M., Van der Schaar, W., Zhuping, Y. and Rajaram, S. (1996) Inheritance of resistance to scab in two wheat cultivars from Brazil and China. Plant Dis. 80, 863-867.

Wang, Y.Z. and Miller, J.D. (1988) Effects of F. graminearum metabolites on wheat tissue in relation to Fusarium head blight resistance. J. Phytopathol. 122, 118-125.

Yanofsky, M.F., Ma, H., Bowman, J.L., Drews, G.N., Feldmann, K.A. and Meyerowitz, E.M. (1990) The protein encoded by the Arabidopsis homeotic gene agamous resembles transcription factors. Nature, 346, 35-39. 